\title{
Questes
}

\section{Le Doute de Joseph devant le miracle de la Conception (Matthieu, I, 18-20)}

\section{Simon Gabay}

\section{OpenEdition \\ Journals}

\section{Édition électronique}

URL : https://journals.openedition.org/questes/1233

DOI : 10.4000/questes. 1233

ISSN : 2109-9472

\section{Éditeur}

Les Amis de Questes

\section{Édition imprimée}

Date de publication : 15 mars 2012

Pagination : 85-103

ISSN : 2102-7188

\section{Référence électronique}

Simon Gabay, «Le Doute de Joseph devant le miracle de la Conception (Matthieu, I, 18-20) », Questes

[En ligne], 23 | 2012, mis en ligne le 01 janvier 2014, consulté le 21 septembre 2021. URL : http:// journals.openedition.org/questes/1233 ; DOI : https://doi.org/10.4000/questes.1233 


\section{Le Doute de Joseph devant le miracle de la Conception \\ (Matthieu, I, 18-20) \\ Simon GABAY}

Si la question de l'existence d'un doute philosophique au Moyen Âge, tel que celui qui, manifesté par les diverses formes du scepticisme, interroge la possibilité de connaître ${ }^{1}$, a été résolue par l'affirmative, celle de l'existence d'un doute théologique semble encore dans les limbes de la recherche. Les miracles de Jésus et des saints, points d'orgue du surnaturel religieux, ont pourtant toujours été l'objet d'un refus manifesté par l'hérésie, l'incroyance ${ }^{2}$ ou tout simplement la peur de la supercherie $^{3}$. Or, sauf à tomber dans un manichéisme qui opposerait simplement l'acceptation au rejet, il nous semble impossible de ne pas admettre l'existence d'une zone grise, dont nous allons tenter ici de démontrer l'existence : le doute quant à la foi.

Il est acquis que le miracle a toujours été suspect, non en fonction d'une de ses formes particulières ${ }^{4}$ mais de sa proximité : on doute toujours

\footnotetext{
${ }^{1}$ Le récent livre de Dominik PerLER (Zweifel und Gewissheit : Skeptische Debatten im Mittelalter, Francfort-sur-le-Main, Vittorio Klostermann, «Philosophische Abhandlungen », 92, 2006), à la bibliographie étoffée, est l'une des meilleures portes d'entrée à l'étude du doute, ainsi que celui de Sabina FlanAGAN (Doubt in an Age of Faith : Uncertainty in the Long Twelfth Century, Turnhout, Brepols, «Disputatio », 17, 2008).

${ }^{2}$ Christian CANNUYER résume la question et donne une bibliographie élémentaire dans son article «Des incroyants au Moyen Âge? », Mélanges de science religieuse, 63, 3 (2006), p. 21-32.

${ }^{3}$ Le montre l'exemple de San Ciappelletto, le pire des chrétiens devenu saint homme en trompant éhontément un moine, et dont la dépouille finit par passer pour miraculeuse auprès d'une population locale bernée. Cf. BocCACE, Décaméron, Journée I, Nouvelle I. ${ }^{4}$ Le miracle supra naturam suscite par exemple plus de doute que celui extra ou contra naturam, si l'on reprend la division thomiste. Cf. THOMAS D'AQUIN, De potentia, q. 6 a. 2 sol. 3. Pour les références aux œuvres de Thomas d'Aquin, nous utilisons ici et infra l'édition électronique des Opera omnia réalisée par Enrique ALARCón et disponible sur www.corpusthomisticum.org.
} 
plus d'un miracle temporellement proche que d'un miracle lointain ${ }^{5}$, preuve d'une réticence instinctive à la nature même de ce phénomène ${ }^{6}$. La théologie médiévale questionne vigoureusement le miracle dans le cadre du débat provoqué par l'intégration d'Aristote en Occident, et il a été prouvé que la position thomasienne (dans laquelle le miracle est, contrairement à ce qu'en pense Augustin, un « argument rationnel imparable » ${ }^{7}$ ) s'élabore contre les thèses condamnées des artiens de l'Université de Paris qui rendent le miracle philosophiquement impensable ${ }^{8}$. Ces fidéistes rejettent hors du champ rationnel ce phénomène divin qui devient presque un obstacle (car scientifiquement inexplicable) à la croyance : je ne crois pas grâce au miracle (il ne confirme pas la foi), mais j'accepte le miracle parce que je crois (il n'est qu'objet de foi). Paradoxalement pourtant, le débat chez les historiens de la religion concernant l'adhésion de l'homme aux éléments surnaturels du christianisme tend à prendre comme point de départ parfois la Renaissance, mais parfois encore le rationalisme allemand et sa critique historico-scientifique ${ }^{9}$, omettant un Moyen Âge toujours inconsciemment compris comme cet « empire du Christ ${ }^{10}$ monolithique.

Une époque si ancrée dans la croyance peut-elle douter des miracles ? La question ne devrait pas se poser, tant la présence du doute est

\footnotetext{
${ }^{5}$ Cf. Max SECKLER, «Plädoyer für Ehrlichkeit im Umgang mit Wundern », Tübinger Theologische Quartalschrift, 151 (1971), p. 337-345.

${ }^{6}$ Benoît Bourgine en langue française ( $«$ Le miracle dans la théologie fondamentale classique », Recherches de science religieuse, 98 (2010), p. 497-524) et Michael MAHER en langue anglaise ( «Recent writings on the Miracles », New Blackfriars, 56 (2007), p. 165-174) résument les grandes évolutions de cette question.

${ }^{7}$ Cf. Gilles BerCEVILLE, «Les miracles comme motifs de crédibilité chez Thomas d'Aquin », Mélanges de science religieuse, 53, 1 (1996), p. 52.

${ }^{8}$ Cf. François Pouliot, La Doctrine du miracle chez Thomas d'Aquin: Deus in omnibus intime operatur, Paris, Vrin, «Bibliothèque thomiste », 56, 2005, p. 32 et sqq. (en particulier p. 137).

${ }^{9}$ Centrée sur la France, l'étude de François LAPLANChE (La Bible en France entre mythe et critique (XVI ${ }^{\mathrm{e}}-\mathrm{XIX} \mathrm{X}^{\mathrm{e}}$ siècle), Paris, Albin Michel, «L'Évolution de l'humanité », 1994) reste une excellente introduction à la matière.

${ }^{10}$ La formule est de Jacques MARITAIN dans Humanisme intégral, Paris, Aubier, 1936, p. 166.
} 
manifeste dans les Évangiles mêmes : saint Thomas n'a-t-il pas douté de la Résurrection $^{11}$ (et de l'Assomption ${ }^{12}$ ), tout comme Jean le Baptiste ${ }^{13}$ ? Et saint Pierre n'a-t-il pas douté de la Marche sur les eaux ${ }^{14}$ ? Cette anticipation du doute du fidèle dans le texte biblique même se trouve d'ailleurs explicitée par les théologiens là où elle est moins évidente encore: l'Aquinate explique ainsi que les quarante jours que durent la Résurrection servent à éviter l'idée d'une illusion ${ }^{15}$, il débat aussi de la question d'un éventuel doute de Marie lors de la mort du Seigneur ${ }^{16}$ et même du Christ («en tant qu'homme » uniquement) sur la Croix ${ }^{17}$ !

Comment comprendre alors l'importance de la réception à la fois théologique et populaire ${ }^{18}$ de ces passages bibliques, et plus généralement du doute devant le miracle au Moyen Âge ? Plutôt que d'accumuler des exemples, nous nous proposons ici de nous attarder sur un seul cas : celui de Joseph, qui ne comprend pas la maternité miraculeuse de Marie, devant la Conception virginale du Christ. Premièrement, ce miracle n'entre pas dans la catégorie de ceux qui confirment la foi (ad fidei comprobationem),

${ }^{11}$ Sur Jean, XX, 24-29, voir ThOMAS D'AQUIN, Scriptum super Sententiis, lib. 3 d. 21 q. 2 a. 4 qc. 3 ad 3 : «Tetigit eum, ut a nobis omnem dubitationem excluderet» («Il l'a touché pour écarter de nous tout doute ». Nous traduisons). Sur cet aspect de l'apôtre, voir Glenn W. Most, Doubting Thomas, Cambridge/Londres, Harvard University Press, 2005.

${ }^{12} \mathrm{Ce}$ second doute est souvent oublié, bien que présent au Moyen Âge comme en témoigne la Sainte Ceinture du dôme de Prato (donnée par la Vierge à l'apôtre Thomas, qui ne voulait pas croire à son Assomption). Voir Claude et François PARFAICT, Histoire du théâtre françois, depuis son origine jusqu'à présent, Paris/Beauvais, Le Mercier/Saillant, 1745, vol. 3, p. 79-80 pour une version théâtrale de ce miracle.

13 À propos de Matthieu, XI, 2-6, voir ThOMAS D'AQUIN, Scriptum super Sententiis, lib. 3 d. 25 q. 2 a. 2 qc. 3 ad 1.

${ }^{14}$ Cf. Matthieu, XIV, 27-31.

15 Thomas D'AQuin, Scriptum super Sententiis, lib. 3 d. 22 q. 3 a. 2 qc. 3. Cf. Matthieu, XIV, 31 et XXVIII, 17.

${ }^{16}$ Le point est soulevé par ThOMAS D'AQUIN (Scriptum super Sententiis, lib. 3 d. 3 q. 1 a. 2 qc. 2 arg. 1) avant d'être démenti (ibid., lib. 3 d. 3 q. 1 a. 2 qc. 2 ad 1).

17 THOMAS D'AQUIN, Scriptum super Sententiis, lib. 3 d. 17 q. 1 a. 4.

${ }^{18}$ Nous avons exposé ailleurs l'importance d'une confrontation de ces deux approches. Cf. Simon GABAY, «Le statut juridique de l'acteur en droit canon au Moyen Âge », in Le Théâtre de l'Église, Paris, Marie BOUHAÏK-GIRONÈS et Dominique IOGNA-PrAT (dir.), LAMOP, 2011 ( 1 ère éd. en ligne 2011). 
mais de ceux qui sont objets de foi (de quibus est fides) ${ }^{19}$ et donc intrinsèquement liés à l'Incarnation rédemptrice (le «Miracle des miracles » thomasien ${ }^{20}$ ), lui conférant une centralité certaine dans la théologie catholique. Sans nécessairement être représentatif, il reste néanmoins à nos yeux assez essentiel pour justifier notre attention. Deuxièmement, il concerne un doute typiquement médiéval : presque né à cette époque sous la plume d'Hugues de Saint-Cher ${ }^{21}$, il va disparaître à la Renaissance avec le concile de Trente $^{22}$, mais connaît entre temps une popularité et une importance certaines, puisqu'il est mis au même rang que les miracles précédemment cités :

[...] ainsi que nous sommes mieux assurés de la résurrection du Christ par Thomas touchant les plaies du Christ que par d'autres, nous sommes mieux assurés de la virginité de Marie par Joseph $[\ldots]$. $^{23}$

Oublié aujourd'hui ${ }^{24}$, cet épisode reste un élément important de la religiosité médiévale, dont il présente, dans son origine et son expression théologique et artistique, bon nombre de caractéristiques. Cette expression artistique va ainsi nous permettre de dépasser une simple approche du doute dans la littérature érudite, au profit d'une étude du sentiment religieux.

\footnotetext{
${ }^{19}$ ThOMAS D'AQUIN, Somme théologique, IIIa q. 29 a.1 ad. 2. Sur cette distinction, voir Gilles BERCEVILLE, «L'étonnante Alliance : Évangile et miracles selon saint Thomas d'Aquin », Revue thomiste, 103, 1 (2003), p. 43.

${ }^{20}$ Ibid., p. 41.

${ }^{21}$ Cf. Paul PayAn, Joseph. Une image de la paternité dans l'Occident médiéval, Paris, Aubier, 2006, p. 83. La théologie d'Hugues de Saint-Cher est discutée infra.

${ }^{22}$ Cf. Louis RÉAU, Iconographie de l'Art Chrétien, II. Iconographie de la Bible, Paris, PUF, 1957, p. 207.

${ }^{23}$ NiCOLAS DE LYRE, Biblia sacra cum Glossa interlineari, ordinaria, et Nicolai Lyrani Postilla, Venise, 1588, $\mathrm{f}^{\circ} 7 \mathrm{v}^{\circ}$ (cité et traduit par Paul PAYAN, Joseph. Une image de la paternité, op. cit., p. 99).

${ }^{24}$ Une trop rapide étude lui a été consacrée par Guy-Marie BERTRAND dans sa thèse de doctorat Saint Joseph dans les écrits des Pères, Cahiers de Joséphologie, 14 (1966), p. 85-101.
} 
Afin de satisfaire à ce but, nous nous proposons, en plus d'une analyse des Évangiles canoniques et apocryphes, de confronter la théologie avec la littérature théâtrale sacrée ${ }^{25}$, et notamment la Passion d'Arnoul Gréban $^{26}$ (rédigée vers $1450^{27}$ ). Comme tous les autres, ce récit de la vie du Christ ne peut s'affranchir d'une réflexion théologique à la fois dépendante des sources universitaires (la censure guette ${ }^{28}$ ), et d'une autre source qui lui est propre: cette pièce de théâtre n'est ni la messe, ni un cours de la Sorbonne, mais un spectacle qui obéit à ses propres règles, comme celles de son succès nécessaire (le retour sur investissement est obligatoire, le profit souhaité ${ }^{29}$ ) ou sa performativité (ce n'est pas un texte, mais un jeu ${ }^{30}$ ). Nous espérons que le traitement réservé à Joseph par Arnoul Gréban permettra ainsi de rentrer plus en profondeur et d'une manière originale dans la civilisation médiévale.

\section{Origines scripturaires et apocryphes du Doute de Joseph}

L'histoire du Doute de Joseph prend sa source dans l'Évangile de Matthieu, au moment du retour de Marie de chez sa cousine Élisabeth :

\footnotetext{
${ }^{25}$ Sur les Passions médiévales, le livre de Jean-Pierre BORDIER (Le Jeu de la Passion. Le message chrétien et le théâtre français $\left(\mathrm{XIII}^{\mathrm{e}}-\mathrm{XVI}^{\mathrm{e}}\right.$ siècle), Paris, Champion, «Bibliothèque du $\mathrm{XV}^{\mathrm{e}}$ siècle », 1998) reste la meilleure étude récente.

${ }^{26}$ L'édition de référence, bien qu'insuffisante, est Le Mystère de la Passion d'Arnoul Gréban, Omer JoDOGNE (éd.), Bruxelles, Palais des Académies, 2 vol., 1965-1983. Une version plus accessible reste celle traduite et abrégée, mais omettant l'épisode du Doute de Joseph, de Jean SubrenAt, Le Mystère de la Passion, Paris, Gallimard, «Folio », 1987.

${ }^{27}$ Cf. Graham A. Runnalls, «The Linguistic Dating of Middle French Texts with Special Reference to the Theatre », Modern Language Review, 71 (1976), p. 757-765.

${ }^{28}$ Cf. Graham A. RunNALls, « Sponsorship and Control in Medieval French Religious Drama : 1402-1548 », French Studies, 51, 3 (1997), p. 257-266.

${ }^{29}$ L'étude menée par Matthieu BonICEL dans les registres de comptes (Arts et gens du spectacle à Avignon à la fin du Moyen Âge (1450-1550) d'après les archives communales d'Avignon, Paris, École nationale des chartes, 2006) montre bien cette dimension financière de la performance.

${ }^{30}$ Le livre d'Elaine Aston et George SAVOnA (Theatre as Sign-system. A Semiotics of Text and Performance, Londres/New York, Routledge, 1991) n'est qu'une introduction, mais a le mérite de poser clairement la question de la relation entre texte et performance.
} 
[...] avant qu'ils eussent mené vie commune, elle se trouva enceinte par le fait de l'Esprit Saint.

Joseph, son mari, qui était un homme juste et ne voulait pas la dénoncer publiquement, résolut de la répudier sans bruit. Alors qu'il avait formé ce dessein, voici que l'Ange du Seigneur lui apparut en songe et lui dit: «Joseph, fils de David, ne crains pas de prendre chez toi Marie, ta femme : car ce qui a été engendré en elle vient de l'Esprit Saint. ${ }^{31}$

Une première remarque s'impose : comme on peut le voir, le récit de Matthieu ne mentionne aucun doute. Puisque le seul autre évangile racontant la naissance du Christ omet le passage ${ }^{32}$, c'est donc vers les sources apocryphes, ces livres «tombé[s] dans le domaine des connaissances courantes $»^{33}$, qu'il faut se tourner. Tentant d'assouvir la soif de connaissance des chrétiens à propos de la personne du Christ, elles vont «broder » sur la source néo-testamentaire (notamment l'enfance qui y est peu traitée) et faire apparaître l'épisode du Doute de Joseph.

L'Histoire de Joseph le Charpentier propose ainsi le début d'un doute, en montrant un Joseph qui «fut troublé dans son esprit» et qui « songeait à renvoyer [Marie] en secret ${ }^{34}$. L'auteur ne poursuit cependant pas plus cette idée, qui est véritablement développée de manière plus innovante dans le Pseudo-Matthieu. Ce dernier ajoute à la scène des jeunes filles entourant Marie lorsque Joseph la découvre enceinte (ils ont été séparés neuf mois) ${ }^{35}$ : elles tentent d'anticiper sa réaction («Vraiment, si tu veux que nous te dévoilions nos soupçons, personne ne l'a rendue enceinte si ce n'est l'ange de Dieu. ${ }^{36}$ ), mais ce dernier ne les croit pas et penche

\footnotetext{
${ }^{31}$ Matthieu, I, 18-20. Nous utilisons la traduction de la Bible de Jérusalem.

${ }^{32}$ Luc, I, 24. Rappelons que les Évangiles de Jean et Marc commencent après la naissance de Jésus.

33 Expression de Paul ZUMTHOR, Histoire littéraire de la France médiévale, VIe-XIV siècles, Paris, PUF, 1954, p. 99.

${ }^{34}$ Histoire de Joseph le Charpentier, in Évangiles apocryphes, France QUÉRÉ (éd.), Paris, Seuil, «Points. Sagesses », 34, 2004, chap. V. Nous utilisons cette édition pour tous les évangiles apocryphes.

${ }^{35}$ Pseudo-Matthieu, in Évangiles apocryphes, op. cit., chap. X, 1.

${ }^{36}$ Ibid., chap. X, 1.
} 
pour l'hypothèse réaliste de la tromperie ${ }^{37}$. Ce point appelle deux remarques: d'une part, l'auteur semble vouloir déguiser le doute devant la Conception en externalisant (par les filles) l'une des deux hypothèses contradictoires (l'action d'un ange) afin de présenter un Joseph (qui croit à l'adultère) sûr de lui ; d'autre part, il insinue qu'une voix terrestre (ou tout du moins non autorisée) ne peut annoncer le miracle - on retrouvera cette idée chez Nicolas de Lyre. Le récit rejoint ensuite celui de Matthieu : songeant à répudier sa femme, Joseph ne sera convaincu que par la voix de l'Ange $^{38}$.

C'est le Protévangile de Jacques qui transpose dans un personnage unique l'hésitation entre les deux possibilités sur la paternité de l'enfant, et de ce fait formalise pleinement le doute en le faisant passer de l'échelle du dialogue à celle du questionnement interne. Joseph (après cette fois six mois de séparation) envisage instinctivement une tromperie de Marie ${ }^{39}$ sans que celle-ci, qui se trouve cette fois seule avec lui, ne l'aide à comprendre ${ }^{40}$. Mais, alors que la question de l'origine de la maternité semble être résolue, il revient dessus dans un second temps en la mêlant à la question du sort à réserver à sa femme, pour aboutir à une hésitation double : est-ce une faute ou un mystère ? Dois-je garder le secret ou la dénoncer ?

Et Joseph, rempli de frayeur, se tint coi, et il se demandait ce qu'il devait faire d'elle. «Si je garde le secret sur sa faute, se disait-il, je contreviendrai à la loi du Seigneur. Mais si je la dénonce aux fils d'Israël, et que son enfant vienne d'un ange, ce dont j'ai bien peur, alors je livre à la peine capitale un sang innocent. Que ferai-je d'elle ? Je la répudierai en secret. » La nuit le surprit dans ces réflexions. ${ }^{41}$

\footnotetext{
${ }^{37}$ Ibid., chap. X, 2.

${ }^{38}$ Ibid., chap. XI.

${ }^{39}$ Protévangile de Jacques, in Évangiles apocryphes, op. cit., chap. XIII, 12.

40 «Joseph lui dit: "D'où vient le fruit de ton sein ?" Et elle répondit: "Aussi vrai que vit le seigneur mon Dieu, j'ignore d'où il vient." » (Ibid., chap. XIII, 3).

${ }^{41}$ Ibid., chap. XIV, 1.
} 
Deux conclusions peuvent être tirées de ces premières notes. À propos de l'importance du Doute de Joseph, outre sa faiblesse quantitative dans la masse des écrits canoniques et apocryphes, la chronologie de ces derniers (les trois mentionnés sont respectivement des $\mathrm{IV}^{\mathrm{e}}, \mathrm{VII}^{\mathrm{e}}$ et II $^{\mathrm{e}}$ siècles) ne laisse pas se dégager une évolution tendant à accentuer l'intérêt pour cet épisode biblique, l'apocryphe le plus explicite étant chronologiquement le premier. Concernant sa nature, elle se révèle être complexe : le doute semble se dédoubler entre un premier doute devant l'origine de la maternité de la Vierge et un second devant l'attitude à adopter par Joseph après sa découverte.

\section{Origine exégétique du Doute de Joseph}

Cette articulation en deux temps du Doute de Joseph va se retrouver dans son exégèse, dans la mesure où tous les commentateurs vont admettre l'existence du second doute (que faire ?), mais pas nécessairement du premier (d'où vient cet enfant?). De fortes différences existent en effet entre les théologiens, que la taxinomie de Pierre de Jean Olivi regroupe efficacement en trois pôles ${ }^{42}$.

Plusieurs exégètes considèrent que Joseph a légitimement pu croire la Vierge victime d'un viol. Parmi eux, Justin dans son Dialogus cum Tryphone $^{43}$, Jean Chrysostome dans In Matthaeum ${ }^{44}$, et surtout Augustin dans son cinquante-et-unième sermon : «Quia enim de illo gravidam non esse sciebat, iam velut consequenter adulteram existimabat $»^{45}$. Comme le

\footnotetext{
${ }^{42}$ Cf. Aquilin EMmEN, «Pierre de Jean Olivi, sa doctrine et son influence », Cahiers de Joséphologie, 14 (1966), p. 242-244.

43 Justin De NAPlouse, Dialogus cum Tryphone Judaeo, Jacques-Paul Migne (éd.), Petit-Montrouge, «Patrologia Graeca Cursus Completus», 6, 78, 3, col. 657. La «Patrologia Graeca Cursus Completus » sera désormais abrégée en «PG ».

44 Jean Chrysostome, In Matthaeum homiliae, Jacques-Paul Migne (éd.), Petit-Montrouge, «PG », 58, Hom. 4, 7, col. 48.

45 «Étranger à cette conception, il en concluait quelle était adultère. » (AUGUSTIN D'HipPone, Sermones ad populum. Classis I. De scripturis, Jacques-Paul Migne (éd.),
} 
montre le récit du Pseudo-Augustin, le doute ne porte que sur la décision à prendre par Joseph qui, « turbatur ${ }^{46}$, hésite :

Si prodidero, adulterio non consentio, sed vitium crudelitatis incurro quia secundum Moysi sententiam lapidandam eam esse cognosco. Si tacuero, malo consentio et cum adulteris portionem meam pono. $^{47}$

Cependant, une seconde tradition veut que Joseph ne fut pas sujet au doute, mais fut effrayé par la grandeur du mystère divin, comme l'explique saint Jérôme :

Sed hoc testimonium Mariae est quod Ioseph, sciens illius castitatem et admirans quod euenerat, celat silentio cuius mysterium nesciebat. ${ }^{48}$

Cette tension chez Jérôme entre la découverte du mystère ${ }^{49}$ et le maintien de la confiance en Marie se retrouve aussi chez Basile de Césarée ${ }^{50}$, Pierre Chrysologue $^{51}$ et enfin un Pseudo-Origène du $\mathrm{VI}^{\mathrm{e}}$ siècle ${ }^{52}$ qui pense que

Petit-Montrouge, «Patrologia Latina Cursus Completus », 38, Sermo 51, 6, 9, col. 338bc. Nous traduisons). La «Patrologia Latina Cursus Completus » sera désormais abrégée en «PL ».

46 «[...] secumque aestuabat disputans et dicens : quid faciam ? Prodo aut taceo ?» («Ces pensées l'agitent tour à tour et se confondent dans son esprit: Que ferai-je ? Dois-je faire connaître son crime ou me taire?», PSEUDO-AUGUSTIN (Attribué à Augustin), Sermones suppositi. Classis III. De sanctis, Jacques-Paul Migne (éd.), Petit-Montrouge, «PL », 39, Sermo 195, 4, col. 2108. Nous traduisons).

47 «Si je dévoile sa faute, je proteste contre l'adultère, mais je m'expose au reproche de cruauté, car je sais que d'après la loi de Moïse elle doit être lapidée. Si je garde le silence, je me rends complice du mal, et je fais alliance avec les adultères » (ibid. Nous traduisons).

48 «Mais voilà un témoignage en faveur de Marie : il savait qu'elle était chaste et il était surpris de ce qui était arrivé, il cachait par son silence ce dont il ne comprenait point le mystère » (JÉRÔME DE STRIDON, Commentaire sur l'Évangile de Matthieu, t. I, Paris, Éditions du Cerf, «Sources Chrétiennes », 242, 1978, In Matthaeum, I, 19, p. 78-79).

49 JÉrôMe De Stridon, De perpetua Virginitate Beatae Mariae, Jacques-Paul Migne (éd.), Petit-Montrouge, «PL», 23, 1, 4, col. 196a: «Joseph, qui sponsae uterum tumentem pene jam licentia maritali, et curiosis oculis deprehendit» («Joseph, qui regardait le ventre rond de son épouse, d'un regard anxieux ». Nous traduisons).

${ }^{50}$ BASILE DE CÉSARÉE, Homilia in sanctam Christi generationem, Jacques-Paul Migne (éd.), Petit-Montrouge, «PG», 31, col. 1463-1466. Texte traduit et commenté par G. M. BERTRAND, Saint Joseph dans les écrits des Pères, op. cit., p. 87.

51 Pierre Chrysologue, Sancti Petri Chrysologi Collectio sermonum, Alexandre Olivar (éd.), Turnhout, Brepols, vol. 2, «Corpus chrisianorum. Series latina », 24B, 1982, Sermo 145, 1, p. 889-891. 
Joseph a reconnu la prophétie d'Isaï ${ }^{53}$. Certains, comme Eusèbe de Césarée $^{54}$, développent cette idée et imaginent que Joseph, par une intuition ou une révélation surnaturelle, connaissait au moins en partie le mystère et se croyait indigne d'y participer. Saint Rémi s'appuie sur la reconnaissance de la prophétie de Isaïe, XI, 1 par Joseph («[...] non diffidebat hanc prophetiam in ea esse implendam [...] ${ }^{55}$ ), tout comme Bède le Vénérable (qui soutient même l'idée d'un raisonnement personnel au détriment de l'ange $^{56}$ ), ou Raban Maur ${ }^{57}$. Saint Bernard perpétue cette tradition exégétique ; il reprend Jérôme et le Pseudo-Origène ${ }^{58}$ : le doute devient sous sa plume, par le biais des comparaisons entre Pierre et le centurion, une expression des scrupules de l'homme.

52 PSEUdO-ORIGÈnE (attribué à Paul Diacre), Homilia de tempore, Jacques-Paul Migne (éd.), Petit-Montrouge, «PL », 95, col. 1164 et PSEUDO-ORIGÈNE, Origines' Werke : Origenes Matthäuserklärung, E. BENZ et E. KLOSTERMANN (éd.), Berlin, AkademieVerlag, «Die Griechischen Christlichen Schriftsteller der ersten Jahrhunderte », 41, 1, 1941, Homélie I sur Matthieu, I, 18-25, p. 241. Sur la célébrité de ce texte, voir Paul PAYAN, Joseph. Une image de la paternité dans l'Occident médiéval, op. cit., p. 398, n. 2 et saint Bernard (cf. infra).

53 «Un rejeton sortira de la souche de Jessé, un surgeon poussera de ses racines » (Isaïe, XI, 1).

54 EusÈBE DE CÉSARÉE, Quaestionnes Evangelicae, Jacques-Paul MignE (éd.), Petit-Montrouge, «PG», 22, 1, 3, col. 883. Texte traduit et commenté par G. M. BERTRAND, Saint Joseph dans les écrits des Pères, op. cit., p. 86-87.

55 «[...] il ne doutait pas que cette prophétie n'eût reçu en elle son accomplissement [...]» (RÉMI D'AUXERRE, Homiliae duodecim, Jacques-Paul Migne (éd.), Petit-Montrouge, «PL », 131, Hom. 4, col. 887b. Nous traduisons).

56 BÈDE LE VÉNÉRABLE, Opera homiletica, D. HuRST (éd.), Turnhout, Brepols, «Corpus Christianorum. Series Latina », 122, 1955, Hom. 1, 5, p. 33. Cf. Georges Ponton, «Saint Joseph d'après l'œuvre de Bède Le Vénérable », Cahiers de Joséphologie, 19 (1971) p. 202.

57 Raban MAUR, Commentarium in Mattheum, Jacques-Paul Migne (éd.), Petit-Montrouge, «PL », 107, col. 749. Cf. Gérald de BECKER, «Raban Maur et la théologie de saint Joseph », Cahiers de Joséphologie, 19 (1971), p. 264.

${ }^{58}$ Cf. Ignazio M. CALABUig, «Les sources patristiques de la pensée mariale de saint Bernard », in Jean LONGÈRE (dir.), La Vierge dans la tradition cistercienne, Paris, Médiaspaul, «Études mariales », 1999, p. 67-68. Pour la référence à Jérôme et au Pseudo-Origène chez Bernard, voir Bernard De ClairvauX, Sermones, J. LeClerCQ, H. Rochais, C.H. Talbot (éd.), Rome, Ed. Cistercienses, « Sancti Bernardi Opera », 4, 1966, Homilia super Missus est 2, 14, p. 31-32. Sur le rapprochement entre les deux textes, voir E. BOISSARD, «Saint Bernard et le Pseudo-Aréopagite », Recherches de théologie ancienne et médiévale, 26 (1959), p. 219-220. 
Une troisième et dernière attitude, qui ajoute au doute d'une éventuelle répudiation de Marie celui de l'origine de sa grossesse, nait dans la tradition exégétique avec Hugues de Saint-Cher ${ }^{59}$, et « représente Joseph comme un homme tiraillé entre deux constatations apparemment incompatibles : la grossesse de Marie, et son éminente sainteté qu'il ne met pas en doute $»^{60}$. Elle est reprise par Pierre de Jean Olivi ${ }^{61}$, Ubertin de Casale $^{62}$ et Nicolas de Lyre, tous trois franciscains. Le premier tire plusieurs conclusions de cette analyse: le grand miracle de l'origine virginale est plus croyable, la sainteté de Joseph est plus convaincante, sa foi grandit et se fortifie, son doute donne une connaissance plus profonde des voies de la Grâce et de la Providence, la vertu de Marie se manifeste plus clairement, le doute préfigure plusieurs mystères à venir ${ }^{63}$. Nicolas de Lyre offre lui aussi plusieurs justifications de ce doute :

Joseph pensant à répudier Marie et qui, assuré du mystère par l'Ange, l'accepte comme sa dame, symbolise l'homme doutant de la foi ou des mœurs, qui est conforté dans la foi par les prédicateurs et les confesseurs, et qui se soumet à eux car ils sont fidèles à l'orthodoxie. ${ }^{64}$

Pour argumenter son propos, Nicolas relève d'ailleurs un point intéressant : la Vierge, qui attend une intervention divine, n'a pas donné la solution à

59 James J. DAVIS, «Saint Joseph in the Postillae of Hugh of St. Cher », Cahiers de Joséphologie, 19 (1971), p. 299-300 (éd. partielle des Postillae et trad. en anglais).

${ }^{60}$ Paul PAYAN, Joseph. Une image de la paternité dans l'Occident médiéval, op. cit., p. 83.

${ }^{61}$ Pierre de Jean Olivi, Postilla super Matthaeum, 1, cité dans A. EMMEN, « Pierre de Jean Olivi, sa doctrine et son influence », art. cit., p. 239-250.

${ }^{62}$ Ubertin de CASAle, Arbor vita, lib. I, c. 10, cité dans P. AlCÁntara Martínez, «La josefologia de Ubertino de Casale», Cahiers de Joséphologie, 19 (1971), p. 345-346.

${ }^{63}$ Cf. A. EMmEN, «Pierre de Jean Olivi, sa doctrine et son influence », art. cit., p. 247-249.

${ }^{64}$ NiCOLAS DE LYRE, Biblia sacra cum Glossa interlineari, ordinaria, et Nicolai Lyrani Postilla, Venise, 1588, $\mathrm{f}^{\circ} 7 \mathrm{v}^{\circ}$ (cité et traduit par Paul PAYAN, Joseph. Une image de la paternité dans l'Occident médiéval, op. cit., p. 100). 
Joseph, le laissant pendant l'intervalle dans un doute devenu presque nécessaire ${ }^{65}$.

Si la théologie voit elle aussi un doute dans Matthieu, I, 18-20, elle semble déplacer la question, posée par les apocryphes, du Doute de Joseph envers sa femme vers celle du Doute de Joseph envers lui-même. De surcroît, elle n'admet que minoritairement le doute devant la maternité, et tend à se concentrer sur l'hésitation concernant la décision à prendre par Joseph.

\section{Le Doute de Joseph dans l'art et chez Arnoul Gréban}

La culture médiévale, profondément touchée par les débats théologiques comme par la culture des apocryphes ${ }^{66}$, ne peut donc que se saisir de ce motif du Doute de Joseph, à propos duquel il cache mal son étonnement, dès lors qu'elle y est confrontée :

Mais comment pourra-ce estre voir

C'une vierge puis concevoir

Et vierge pucelle enfanter?

C'est un point qui fait à doubter

Trop malement. ${ }^{67}$

Il serait trop long d'en recenser toutes les manifestations, et d'analyser toutes les formes dans la littérature et les arts. Concernant sa forme plastique $^{68}$, on peut mentionner le Holkham Bible Picture Book $(1325)^{69}$,

${ }^{65}$ Le texte latin et traduit en anglais se trouve dans J. B. ALLEN et T. A. MoriTZ, A Distinction of Stories. The Medieval Unity of Chaucer's Fair Chain of Narratives for Canterbury, Columbus, Ohio State University Press, 1981, p. 208, n. 33.

${ }^{66}$ Cf. Edina BozÓKY, «Les apocryphes bibliques », in Pierre RichÉ et Guy LOBRICHON (dir.), Le Moyen Âge et la Bible, Paris, Beauchesne, 1984, p. 430-448.

67 «Un Miracle de Saint Valentin», in Théâtre français au Moyen Âge, L. J. N. MonmerQuÉ et F. MiChel (éd. et trad.), Paris, H. Delloye, 1839, p. 307.

${ }^{68}$ Paul PAYAN (Joseph. Une image de la paternité dans l'Occident médiéval, op. cit., p. 45) renvoie à Brigitte HEuBlEIN, Der "verkannte" Joseph. Zur Mittelalterlichen Ikonographie des Heiligen im deutschen und niederländischen Kulturraum, Weimar, VDG, 1998, p. 30-33. On ajoutera à cette référence l'article de Zsuzsa URBACH, " "Dominus possedit me..." (Prov. 8,22). Beitrag zur Ikonographie des Josephszweifels », Acta historiae artium Academiae Scientiarum Hungaricae, 20 
qui montre Joseph touchant le ventre de Marie et fait clairement référence au geste identique d'Élisabeth lors de la Visitation de la Vierge ${ }^{70}$, un sarcophage du musée Crozatier du Puy (IV ${ }^{\mathrm{e}}$ siècle) ${ }^{71}$, l'évangéliaire de Lorsch (vers 810$)^{72}$, la Visitation et épisodes de la vie de la Vierge du Livre d'heures à l'usage de Paris (vers 1410) ${ }^{73}$, une clef de voûte de la cathédrale de Norwich (milieu du $\mathrm{XV}^{\mathrm{e}}$ siècle) ${ }^{74}$ ou le panneau d'un retable de l'ancien couvent de saint Marc à Strasbourg (1410-1420) ${ }^{75}$.

Si l'on se limite à la littérature théâtrale, les exemples sont tout aussi nombreux et géographiquement variés. On en trouve dans le théâtre français, comme le Mystère de l'Incarnation et de la Nativité ${ }^{76}$ ou la Passion de Troyes ${ }^{77}$, mais aussi un peu partout à l'étranger : en Espagne, avec La Representación del nacimiento de Nuestro Señor de Gómez

(1974), p. 230-233. Plusieurs exemples cités infra dans cet article sont inconnus de ces deux études.

${ }^{69}$ Holkham Bible Picture Book, W. O. HASSALl (éd.), Londres, Dropmore Press, 1954, $\mathrm{f}^{\circ} 12$.

${ }^{70}$ Le Livre d'heures à l'usage de Paris juxtapose même ces deux images (cf. infra). À ce sujet, voir Gertrud SCHILLER, Iconography of Christian Art, I, Greenwich (Conn.), New York Graphic Society, 1971, p. 55-56 et Millard MEISS, French Painting in the Time of Jean de Berry: The Late Fourteenth Century and the Patronage of the Duke, Londres/New York, Phaidon Press, «Studies in the History of the European Art », 2, 1967, t. 2, images 7, 90, 183 et 257.

${ }^{71}$ Ibid., p. 45.

${ }^{72}$ Cf. Paul PAYAN, Joseph. Une image de la paternité dans l'Occident médiéval, op. cit., p. 44.

${ }^{73}$ Ibid., p. 243.

${ }^{74}$ L'image en question est la $10 \mathrm{~g}$ du cahier des reproductions. La liste des autres images se trouve p. viii, et l'analyse de cette série de la cathédrale se trouve dans M. D. ANDERSON, Drama and Imagery in English Medieval Churches, Cambridge, University Press, 1963, III, chap. 7.

${ }^{75}$ Le Doute de Joseph, aujourd'hui conservé au musée de l'Euvre de Notre-Dame de la même ville.

76 Mystère de l'Incarnation et de la Nativité de Notre Sauveur et Rédempteur Jésus-Christ, Pierre LE VERDIER (éd.), Rouen, Société des bibliophiles normands, 1884-1886.

${ }^{77}$ Le Mystère de la Passion de Troyes : Mistere de la Passion Nostre Seigneur, Troyes, XV siècle, Jean-Claude BIBOLET (éd.), Genève, Droz, 1987, p. 175-184. 
Manrique (1412-1490) ${ }^{78}$, en Angleterre avec la scène « Joseph's troubles about Mary » du Cycle de York $^{79}$ ou celle de la douzième pièce des NTown Plays (« Joseph's Doubt About Mary $»)^{80} \ldots$

La critique espagnole ${ }^{81}$ et anglo-saxonne ${ }^{82}$ a eu jusqu'à présent tendance à appliquer une lecture réaliste selon laquelle Joseph représente le mari trompé ; mais, si celle-ci reste légitime, nous pensons qu'elle ne doit pas se faire au détriment d'une lecture théologique effectuée à la lumière des sources présentées ci-dessus. Le public, qui connaît ce thème, est probablement capable de voir ce second aspect ; sinon comment expliquer le traitement exclusivement théologique d'Arnoul Gréban ${ }^{83}$, sauf à dire qu'un très grand nombre de personnes ne comprend rien (et il faut ici rappeler que la conséquente tradition manuscrite ${ }^{84}$, les remaniements nombreux $^{85}$, les multiples représentations ${ }^{86}$ de cette Passion prouvent des répercussions beaucoup plus importantes que celles d'un simple texte) ? La volonté de l'auteur, dans notre cas, ne fait aucun doute ${ }^{87}$, et il est possible

${ }^{78}$ Gómez MANRIQUE, La Representación del nacimiento de Nuestro Señor, Revista Hispánica Moderna, Año 2, 2 (janv. 1936), p. 25.

79 «Joseph's troubles about Mary», in York Mystery Plays : a Selection in Modern Spelling, Richard BEADLE et Pamela M. KING (éd.), Oxford, Oxford Paperbacks, 2009, XIII, p. 56-57, v. 233-235 et 266-268.

80 «Joseph's Doubt About Mary », in The N-Town Play: Cotton MS Vespasian D. 8, Stephen SPECTOR (éd.), Oxford, Oxford University Press, « Early English Text Society. Supplementary Series », 11-12, 1991, XII, p. 125, v. 49-53.

${ }^{81}$ Cf. Stanislaw ZIMIC, «El teatro religioso de Gómez Manrique (1412-1491) », Boletín de la Real Academia Española, 57 (1977), p. 353-400.

${ }^{82}$ Cf. Rosemary Woolf, The English Mystery Plays, Berkeley/Los Angeles, University of California Press, «Campus », 257, 1980, p. 171.

${ }^{83}$ ARnOUl GrÉBAN, Le Mystère de la Passion, éd. cit., v. 4013-4262, et notamment les v. 4121-4178.

${ }^{84}$ Voir la liste des manuscrits dans l'édition Jodogne.

${ }^{85}$ Notamment celui de Jean Michel pour la représentation d'Angers en 1486. Cf. JEAN Michel, Le Mystère de la Passion (Angers, 1486), Omer JoDOGNE (éd.), Gembloux, J. Duculot, 1959.

${ }^{86}$ En 1473 , le texte a déjà été représenté trois fois à Paris, et une fois à Abbeville en 1452.

87 Arnoul Gréban est maître ès-arts et bachelier en théologie. Cf. Darwin SMITH, «Arnoul Gréban et l'expérience théâtrale ou l'universitaire naissance des mystères », in X. LEROUX (dir.), Vers une poétique du discours dramatique au Moyen Âge. Actes du 
d'imaginer qu'elle vaut aussi pour les autres textes qui décident sciemment d'aborder cet épisode biblique ${ }^{88}$.

Chez Arnoul Gréban, Joseph, dont le « soussy ne se puet deffaire ${ }^{89}$ et dont le cœur est «en fortes doubtes », se dédouble, discutant avec lui-même pour passer en revue les incohérences de la situation, le passage à l'autre lui-même s'opérant toujours par la reprise interrogative du verbe :

Je ne sçay de ton fait la somme.

Sçavoir? Que dis-tu, meschant homme que je suis ? $[\ldots]^{90}$

Se questionnant perpétuellement, il envisage dans un premier temps la tromperie :

[...] il fault qu'elle se soit meffaicte, car de moy n'est il pas venu ; sa promesse n'a pas tenu, elle a rompu son marïaige. ${ }^{91}$

Mais Joseph ne peut remettre en cause l'honorabilité de la Vierge :

Toucheras tu l'onneur de celle qui est la plus doulce pucelle qui dessoublz les cieulx soit regnant $?^{92}$

Il finit par résumer la complexité de la situation :

Dieulx, quel horreur ! Et le creray je?

Nennil, je mens. Encor ne sçay je.

$[\ldots]$

Briefment, je ne sçay que penser. ${ }^{93}$

Colloque international organisé au Palais Neptune de Toulon les 13 et 14 novembre 2008, Paris, Champion, « Babeliana », 14, 2011.

${ }^{88}$ Ou bien refusent de le faire, comme le Joseph de la Passion de Semur, qui ne doute pas : " Or est il fol qui ce ne croit / Que pucelle peust estre mere / Et ung enfant naisse sans pere ! ( (Émile RoY, Le Mystère de la Passion en France du XIV ${ }^{\mathrm{e}}$ au XVI ${ }^{\mathrm{e}}$ siècle, Paris, Champion, 1904, t. I, p. 43, v. 2166-2168).

${ }^{89}$ ARNOUL GrÉBAN, Le Mystère de la Passion, éd. cit., v. 4121.

${ }^{90}$ Ibid., v. 4130-4132.

91 Ibid., v. 4135-4138.

${ }^{92}$ Ibid., v. 4142-4144.

93 Ibid., v. 4158-4159 et 4156. 
«Excusatio non petita, accusatio manifesta », dit le proverbe latin : que doit-on comprendre et déduire de ce Doute de Joseph dans la Passion, en ce qui concerne le sentiment religieux? Peut-on y trouver une dimension cathartique, celle d'un peuple chrétien qui dit ses doutes sur scène pour mieux les surmonter dans la vie quotidienne? La réponse est compliquée, car si l'emphase exceptionnelle sur ce passage ne vient pas de nulle part (Arnoul aurait pu choisir la théorie selon laquelle Joseph reconnaît la prophétie d'Isaïe), il ne faut pas surinterpréter le texte de Gréban : le doute a certes une raison d'être théologique, mais il en a aussi une seconde, performative, qui ne révèle rien de la mentalité médiévale. Il ne faut en effet pas minimiser l'importance du Doute de Joseph qui aujourd'hui encore, bien qu'évacué de l'art et de la théologie officiels de l'Église, reste un objet de discussion ${ }^{94}$ et un motif dans l'art populaire ${ }^{95}$. Le public hétéroclite du Moyen Âge doit être tenu en haleine, et Arnoul Gréban ne doit pas le lasser : les deux cent vers (une dizaine de minutes sur scène ${ }^{96}$ ) que durent la scène doivent dans une certaine mesure répondre à ses attentes.

Cependant, le doute est le meilleur moyen d'exploiter la théâtralité, surtout sous la forme d'un monologue comme celui de Joseph, en laissant s'exprimer toutes les qualités d'un acteur qui reçoit seul l'attention, et

\footnotetext{
${ }^{94}$ Une simple recherche sur internet le confirme.

95 Nous pensons par exemple au Ritorno di Giuseppe du cantautore italien Fabrizio DE ANDRÉ sur son disque La Buona novella : «E lo stupore nei tuoi occhi salì dalle tue mani / che, vuote intorno alle sue spalle, / si colmarono ai fianchi dalla forma precisa / d'una vita recente, di quel segreto che si svela / quando lievita il ventre. / E a te, che cercavi il motivo / d'un inganno inespresso dal volto, / lei propose l'inquieto ricordo / tra i resti d'un sogno raccolto » («Et la stupeur monta jusqu'à tes yeux de tes mains / qui, vides autour de ses épaules / se remplirent aux hanches de cette forme précise / d'une vie récente, de ce secret qui se révèle / quand le ventre lève. / Et à toi, qui cherchait une raison / de cette ruse qui n'était pas exprimée par le visage, / elle proposa le souvenir inquiet / recueilli dans le reste d'un rêve ». Nous traduisons).

${ }^{96} \mathrm{Si}$ nous prenons comme base le rythme moyen de mille vers par heure établi par Milman PARRY et Albert B. LORD dans The Singer of Tales, Cambridge, Harvard University Press, « Harvard Studies in Comparative Literature », 24, 1960.
} 
Arnoul Gréban, en bon homme de théâtre, exploite son potentiel dramatique au maximum ${ }^{97}$. Le doute est aussi un double mécanisme de suspense, un crescendo à la fois narratif (il rythme le récit) et théologique (il met en valeur son propre terme, l'acceptation du miracle de la Conception). C'est une esthétisation rigoureuse, un doute qui n'obéit pas aux règles d'écriture de la théologie universitaire mais à sa propre rhétorique, prenant la forme qui lui convient sans perdre la justesse de son propos. Il ne faut ainsi pas assigner au traitement du Doute de Joseph dans la Passion une importance démesurée : il correspond aussi à un choix artistique et théologique indépendant d'une quelconque popularité.

L'influence de Nicolas de Lyre ${ }^{98}$, mais aussi celle du Protévangile, sur le Mystère de la Passion ressortent clairement de l'épisode du doute devant l'origine de la Conception, qui se fait même au détriment du traitement réservé à Marie (qui disparaît presque)! Ce choix diffère grandement de celui effectué par bien des dramaturges, qui mentionnent mais ne montrent pas le Doute de Joseph ${ }^{99}$. Arnoul Gréban s'approprie l'épisode dont il offre une exégèse, certes guidée par ses lectures, mais très personnelle.

La Passion se révèle être un intermédiaire intéressant entre la théologie $^{100}$ et la culture médiévale, ou plutôt un médium ayant une

\footnotetext{
${ }^{97}$ Ces vers ne sont d'ailleurs pas sans rappeler les stances du Cid.

${ }^{98}$ Nous ne sommes pas les premiers à la remarquer. Cf. Émile RoY, Le Mystère de la Passion en France, op. cit., p. vi.

${ }^{99}$ Dans le Cycle de York, Marie dit : «Now grete God he you wisse, / And mende you of your mysse / Of me, what so betyde » («Que Dieu soit avec toi, / Et te retire tes doutes / En moi, quoiqu'il arrive », York Mystery Plays : a Selection in Modern Spelling, op. cit., p. 56-57, v. 233-235. Nous traduisons).

${ }^{100}$ Nous ne sommes bien sûr pas le premier à faire un tel constat. Voir l'introduction d'Émile RoY à son Mystère de la Passion en France du XIV ${ }^{\mathrm{e}}$ au XVI ${ }^{\mathrm{e}}$ siècle, Paris, Champion, 1904, t. I, Anna CoRnAGliotTI, «Apocryphes et mystères », in G. MulleR (dir.), Le Théâtre au Moyen Âge. Actes du II Colloque de la SITM (Alençon, 1977), Montréal, L'Aurore, 1981, p. 67-78, et surtout Jean-Pierre BORDIER, Le Jeu de la Passion. Le message chrétien et le théâtre français $\left(\mathrm{XIII}^{\mathrm{e}}-\mathrm{XVI}^{\mathrm{e}}\right.$ siècle), op. cit.
} 
théologie propre, non pas secondaire (par rapport à la théologie officielle) mais à la fois parallèle (ayant ses réponses à des questions générales), primaire (en terme de popularité) et séculière (car mêlant fortement les laïcs). Obéissant à ses propres règles, elle rend compte à la fois des tendances théologiques universitaires et du sentiment religieux du peuple de son époque, et se révèle être un matériel nécessaire (mais non suffisant) dans l'étude de l'histoire des mentalités.

Dans notre cas, elle exemplifie à merveille l'idée que le Moyen Âge connaît non seulement le doute, mais crée et discute ses propres doutes. Ceux-ci concernent parfois le cœur même d'une foi qui n'est pas nécessairement simple à accepter lorsqu'elle touche au surnaturel, sans pour autant que cela semble poser de problème aux autorités religieuses. Voilà qui souligne l'ouverture d'esprit certaine d'une époque pourtant réputée en être dépourvue. Sans dire que les hommes du Moyen Âge épurent leurs doutes sur scène comme les Grecs l'ont fait avec leurs passions, on ne peut qu'admettre le questionnement public du miracle sous la forme dramatique.

Pour les hommes du Moyen Âge comme pour Joseph, l'intérêt du doute se trouve dans sa dimension transitoire vers le retour à la foi. Tous les exégètes sont ainsi d'accord pour dire que la sérénité de Joseph revient avec le message de l'ange. Théodore d'Ancyre résume clairement ce changement d'attitude ${ }^{101}$, tout comme Ambroise :

Hoc autem opinatus est quod traduceret eam quasi ream, antequam ab angelo moneretur : postea autem quasi fidelis nec dubius virginitatis ejus servavit oraculum. ${ }^{102}$

\footnotetext{
101 «Dès qu'il apprit le mystère, ajoutant foi aussitôt à la parole de l'ange et connaissant avec certitude que l'Emmanuel naissait d'une vierge [...], Joseph ne rompit plus le lien des fiançailles voulant lui aussi être serviteur de ce mystère » (THÉODORE D'ANCYRE, Hom. 5, 7, publiée par M. AuBINEAU, «Une Homélie grecque inédite attribuée à Théodore d'Ancyre sur le baptême du seigneur », Orientalia Christiana Periodica, 26 (1960), p. 229).

102 «Avant l'avertissement de l'ange, il avait pensé à livrer Marie comme coupable ; mais après, en toute fidélité et sans aucun doute sur sa virginité, il accomplit le
} 
Le doute a une résolution programmée, comme l'explique Augustin («Qui ergo humana infirmitate turbabatur, divina auctoritate firmatus est ? ${ }^{103}$ ), et devient presque une preuve de l'existence de Dieu. Mais si sa fugacité vaut pour la majorité, certains irréductibles ne s'en déferont jamais, car la disposition du récepteur reste l'élément central :

[...] Innotescunt autem divina hominibus secundum quod diversimode sunt affecti. Nam illi qui habent mentem bene dispositam, secundum veritatem divina percipiunt. Illi autem qui habent mentem non bene dispositam, divina percipiunt cum quadam confusione dubietatis vel erroris $[\ldots]^{104}$

Par peur de ces «dispositions contraires », le christianisme anticipe le questionnement de ses ouailles, et doute rhétoriquement sur scène pour éviter de douter concrètement dans la vie.

commandement de l'ange » (AMBROISE DE MILAN, De institutione virginis, Jacques-Paul Migne (éd.), Petit-Montrouge, «PL », 16, 5, 40, col. 316. Nous traduisons).

103 «Est-il étonnant que si la faiblesse humaine l'a fait chanceler, il ait été raffermi par une autorité divine ?» (Augustin D'HipPONE, Sermones ad populum. Classis I. De scripturis, Jacques-Paul Migne (éd.), Petit-Montrouge, «PL », 38, Sermo 51, 6, 9, col. 338bc. Nous traduisons).

104 «...] Les réalités divines sont connues des hommes suivant la diversité de leurs dispositions. Car ceux qui ont l'esprit bien disposé perçoivent ces réalités dans leur vérité ; mais ceux qui ont des dispositions contraires les perçoivent avec un mélange de doute ou d'erreur [...] » (ThOMAS D'AQuin, Somme théologique, IIIa q. 55 a. 4 rép. Traduction: ThomAs D'AQUIN, Somme théologique, Albert RAULIN (dir.) et AimonMarie Roguet (trad.), Paris, Éditions du Cerf, 2000, t. 4, p. 401). 